\title{
Impact of Calibration Algorithms on Hypoglycaemia Detection in Newborn Infants Using Continuous Glucose Monitors
}

\author{
Matthew SIGNAL ${ }^{1}$, Aaron LE COMPTE ${ }^{1}$, Deborah L HARRIS ${ }^{2,3}$, \\ Phil J WESTON ${ }^{2}$, Jane E HARDING ${ }^{3}$, J. Geoffrey CHASE ${ }^{1}$ on behalf of the CHYLD Study Group
}

\author{
1. Department of Mechanical Engineering, University of Canterbury, Christchurch, New Zealand \\ 2. Newborn Intensive Care Unit, Waikato District Health Board, New Zealand \\ 3. Liggins Institute, University of Auckland, Auckland, New Zealand
}

\begin{abstract}
Neonatal hypoglycaemia is a common condition that can cause seizures and serious brain injury in infants. It is diagnosed by blood glucose (BG) measurements, often taken several hours apart. Continuous glucose monitoring (CGM) devices can potentially improve hypoglycaemia detection, while reducing the number of BG measurements. Calibration algorithms convert the sensor signal into the CGM output. Thus, these algorithms can have a direct impact on measures used to quantify excursions from normal glycaemic levels. The aim of this study was to quantify the effects of calibration sensor error and non-linear filtering of CGM data on measures of hypoglycaemia (defined as BG $<2.6 \mathrm{mmol} / \mathrm{L}$ ) in neonates. CGM data was recalibrated using an algorithm that explicitly recognised the high accuracy of BG measurements available in this study. Median filtering was also implemented either before or after recalibration. Results for the entire cohort show an increase in the total number of hypoglycaemic events (161 to 193), duration of hypoglycaemia (2.2 to $2.6 \%$ of total data), and hypoglycaemic index (4.9 to $7.1 \mu \mathrm{mol} / \mathrm{L}$ ) after recalibration. With the addition of filtering, the number of hypoglycaemic events was reduced (193 to 131), with little or no change to the other metrics. These results show how reference sensor error and thus calibration algorithms play a significant role in quantifying hypoglycaemia. In particular, metrics such as counting the number of hypoglycaemic events were particularly sensitive to recalibration and filtering effects. While this conclusion might be expected, its potential impact is quantified here, in this case for at-risk neonates for whom hypoglycaemia carries potential long-term negative outcomes.
\end{abstract}

Keywords: hypoglycaemia, continuous glucose monitoring, infants, calibration, blood glucose

\section{INTRODUCTION}

Neonatal hypoglycaemia is a common condition that can cause seizures and permanent brain injury in newborns (Stanley and Baker, 1999). There is a wide range of risk factors thought to result in hypoglycaemia including prematurity, low birth weight (LBW) or high birth weight (HBW), and having a mother with diabetes (Stanley and Baker, 1999). There remains significant controversy regarding the definition of hypoglycaemia, and consequently, the effect it can have on the child's later development (Cornblath et al., 2000, Koh et al., 1988). Diagnosis is typically by blood glucose (BG) measurements. However, BG measurements are often taken several hours apart and hypoglycaemic events between BG measurements can go undetected (Harris et al., 2010). Continuous Glucose Monitoring (CGM) devices provide a continuous estimate of BG concentration and have the potential to improve the detection and diagnosis of hypoglycaemia.

CGM devices were first developed in the 1980's to help individuals with type 1 diabetes manage their glucose levels. The first CGM device to be approved by the Food and Drug Administration (FDA) was the MiniMed Continuous Glucose Monitoring System (CGMS), which was approved for commercial use in 1999 (Klonoff, 2000). The CGMS consists of a small pager-like device that receives information from a sensor inserted into the subcutaneous layer, just beneath the skin (Mastrototaro et al., 2002). The sensor is coated with a glucose oxidase membrane and produces a small electrical current, as glucose in the interstitial fluid is oxidised, that is proportional to the glucose concentration. The monitor provides a value every 5 minutes or 288 measurements per day.

Independent BG calibration measurements, normally using a finger-stick glucometer, are required to convert electrical current into meaningful CGM output. Point of care testing devices developed largely for diabetic patients that use hexakinase enzyme reactions to measure the glucose levels in capillary blood are reported to have errors typically in the range of 2-10\% (Solnica et al., 2003, Roche, 2007, Roche, 2008, Abbott, 2010). After initial calibration, it is usually recommended that CGM devices be calibrated at least 4 times daily (Minimed, 2003). A 10 minute time delay is incorporated into the calibration process for every sample to account for the transport of glucose from the blood to the interstitial fluid (Rossetti et al., 2010). 
The retrospective calibration algorithm reconciling BG measurements with CGMS readings used by the CGMS Systems Solutions Software employs linear regression (Klonoff, 2000). The use of linear regression with multiple calibration BG measurements available could contribute to the CGMS reporting high during hypoglycaemia and reporting low during hyperglycaemia (Mastrototaro et al., 2002). However, it may also balance the impact of large sensor errors in finger stick glucose meters. Thus, important clinical observations such as the occurrence, severity and duration of excursions from normal BG levels may be directly affected by the specific calibration algorithm used and any assumptions on the quality of reference calibration values. Importantly, such biases or errors may have little impact on overall care or treatment choices (Signal et al., 2010).

More recently CGM devices have been used in research settings to evaluate aspects of glucose metabolism in at-risk newborn infants (Harris et al., 2010). In this case, laboratory determinations of BG concentrations using a glucose oxidase method were available for the dataset, and therefore can be assumed to be a "gold-standard" assessment. Alternative calibration and data-processing algorithms can be applied to the CGM readings to take advantage of the high accuracy of available BG readings and compared to the default calibration algorithm. This study explores and quantifies the impact of calibration, calibration sensor error, and non-linear filtering on the incidence and severity of hypoglycaemia in neonates using CGM devices.

\section{SUBJECTS AND METHODS}

\subsection{Subjects}

This study uses CGM data from 50 babies at risk of hypoglycaemia who were admitted to the Waikato Hospital Newborn Intensive Care Unit (NICU). Table 1 presents the cohort demographics for the 50 patients. Demographics are presented as median [interquartile range] where applicable.

Table 1: Patient demographics

\begin{tabular}{|l|c|}
\hline Cohort Demographics & \\
\hline Number of CGM traces & 50 \\
\hline Sex (M/F) & $26 / 24$ \\
\hline Gestational Age (weeks) & $34[33-37]$ \\
\hline Birthweight (g) & $2172[1880-2990]$ \\
\hline Primary Risk (\# infants): & 15 \\
\hline Diabetes & 19 \\
\hline Premature & 14 \\
\hline Small or Large for gestational age & 2 \\
\hline Other & \\
\hline
\end{tabular}

\subsection{Continuous Glucose Monitoring}

All patients had interstitial glucose monitoring using the CGMS ${ }^{\circledR}$ System Gold ${ }^{\mathrm{TM}}$ (Medtronic, Minimed, Northridge, CA, USA). Monitoring began on admission to the NICU and finished after 7 days or when the baby was no longer considered to be at risk of hypoglycaemia, whichever came first. During the monitoring period nurses were asked to record all $\mathrm{BG}$ concentrations, feeding and medication for the management of hypoglycaemia. However, they remained blind to the glucose concentrations determined by the device. The device was calibrated per the manufacturer's recommendations and all of the data entered into the device was checked against clinical records for accuracy. Upon completion of monitoring, data were downloaded to a PC using CGMS system solutions software version 3.0C, which calibrated the CGM readings retrospectively.

\subsection{Calibration Measurements}

All BG calibration measurements were made using a blood gas analyser (Radiometer, ABL800Flex, Copenhagen) using the glucose oxidase method. This device has a reading range of 0.0 to $60.0 \mathrm{mmol} / \mathrm{L}$ and a coefficient of variation of $2.1 \%$ (Harris et al., 2010). Due to the location of the blood gas analyser, a short time delay (estimated $<15$ mins maximum) was possible between taking the blood sample and introducing it into the device.

\subsection{Calibration Algorithms}

The calibration algorithm used by the CGMS is based on linear regression (Mastrototaro et al., 2002, Chee et al., 2001). The algorithm reduces the impact of inherent error in the calibration measurements on the overall accuracy of the device. The linear regression algorithm is aimed primarily at ambulatory individuals with type 1 diabetes who use the CGM device to help manage BG levels. This population typically uses a finger stick glucometer, which analyses capillary BG and typically has up to $10 \%$ measurement error (Roche, 2007, Roche, 2008, Abbott, 2010, Solnica et al., 2003). Hence, the use of linear regression implicitly balances reference sensor and CGMS errors, and CGM outputs do not necessarily exactly correspond to BG measurements.

Figure 1 shows an example of how linear regression is used in the factory calibration. The crosses represent BG calibration measurements plotted against the sensor current $\left(\right.$ Valid_I $\left.I_{S I G}\right)$ sample at the same time, together with the fitted regression line. Crosses above the regression line will result in the CGM reading low at that time point, and crosses below the regression line will result in the CGM reading high.

Since the calibration measurements in this study were determined using a gold standard for BG measurement, the data were recalibrated using the algorithm in Table 2, which was designed to make better use of these more accurate calibration measurements by forcing CGM output through BG measurements. It should be noted that there are many ways that the data could be recalibrated and the algorithm used in this study represents just one example based as directly as possible upon the current method (Klonoff, 2000, Minimed, 2003). 


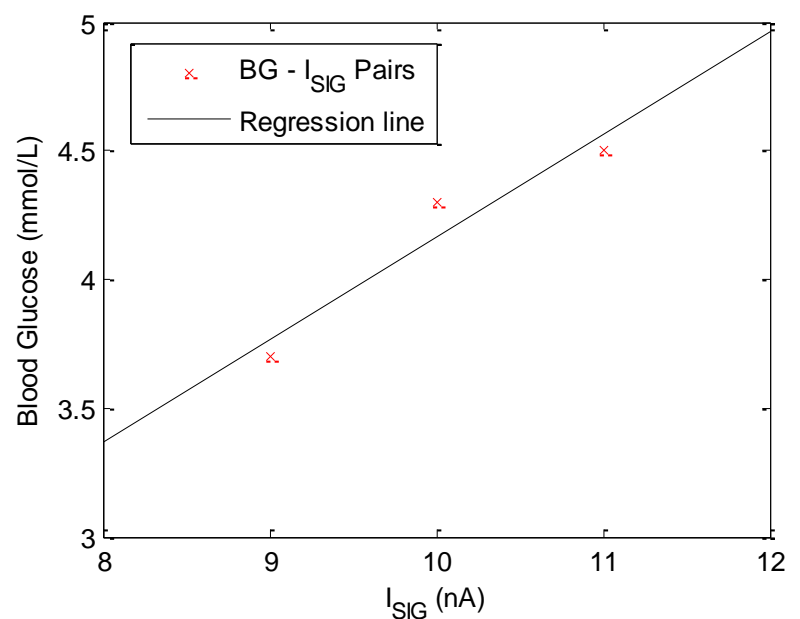

Figure 1: Example of how linear regression is used in the factory calibration. Crosses represent calibration measurements, paired with the sensor current at the same point in time.

Table 2: Recalibration algorithm used with Blood Gas calibration measurements

Factory CGM blood glucose estimation:

$B G_{C G M}=$ Slope $*\left(\right.$ Valid_I $I_{\text {sig }}-$ offset $)$

Where: $\begin{array}{ll}B G_{C G M} & \text { Estimated blood glucose level by the } \\ & \left.\text { CGM (mmol.L }{ }^{-1}\right)\end{array}$

Slope Calibration parameter found using linear regression $\left(\mathrm{mmol} . \mathrm{L}^{-1} / \mathrm{nA}\right)$

Valid_I $I_{S I G} \quad$ Electrical current detected by the monitor from the sensor $(\mathrm{nA})$

Offset Calibration parameter that is used if the sensitivity ratio is below a threshold

To recalibrate, rearrange (1) to:

Reqd_slope $(i)=\frac{B G_{\text {Cal }}(i)}{\left(\text { Valid_I } I_{\text {sig }}(i)-\text { offset }(i)\right)}$

Where:

$B G_{\text {Cal }} \quad$ Blood glucose level for calibration Blood Gas analyser (mmol. $\left.\mathrm{L}^{-1}\right)$

Reqd_slope Slope that forces $\mathrm{BG}_{\mathrm{CGM}}$ to pass through calibration measurements (mmol. $\left.\mathrm{L}^{-1} / \mathrm{nA}\right)$

Linearly interpolate between Reqd_slope(i) and Reqd_slope $(\mathrm{i}+1)$ to obtain a continuous slope function for $\mathrm{i}=1: \mathrm{N}-1 B G_{C a l}$ measurements. Replace Slope in (1) with the slope function from (2) to obtain recalibrated $B G_{C G M}$.
The recalibration algorithm forces the output CGM trace to pass through the calibration BG measurements, while preserving the raw sensor current (Valid_I IIG) and Offset parameter. At each calibration measurement a value of slope (Reqd_slope) is calculated using Equation 2, which will force the CGM to pass through the BG. Linear interpolation between successive values of Reqd_slope gives the new, continuous slope function. The new slope function is inserted to Equation 1 with the unmodified Valid_I $I_{S I G}$ and Offset parameters, to give the recalibrated CGM estimate of BG. Forcing the CGM trace through the calibration measurements recognises explicitly the higher accuracy of the blood gas analyser, and thus provides a comparator to assess the impact of calibration on outcome CGM traces.

\subsection{Median Filtering}

Median filters are used to remove unwanted and potentially un-physiological high-frequency noise from the CGM signal. They have proven to be a simple and effective method of removing this noise and smoothing CGM traces (Pretty et al., 2010). A retrospective composite median filter was used in this study because it allows faster and slower glucose dynamics to be captured more effectively. The filter averages a 3 point median and a 5 point median both centred about the time point of interest. The filter was implemented both prior to recalibration (on the Valid_I $I_{S I G}$ ) and post calibration on the CGM output to test the effect of applying filtering before and after calibration calculations.

\subsection{Analysis}

Four analyses of the CGMS data from the 50 babies were performed in this study: 1) Original CGM output; 2) recalibrated CGM output; 3) re-calibrated CGM output with median filtering on post-calibration readings; and finally, 4) re-calibrated CGM output with median filtering the precalibration sensor current (Valid_I $\left.I_{S I G}\right)$. Each of the recalibrated variations, with and without filtering, is compared to the original CGM output to see the effect of recalibrating/filtering on clinical measures of hypoglycaemia. The metrics used to quantify hypoglycaemia were:

- Number: Number of independent hypoglycaemic events $(\mathrm{CGM}<2.6 \mathrm{mmol} / \mathrm{L})$

- Duration: Percent of CGM record below 2.6mmol/L

- Severity: Lowest measurement of hypoglycaemic event.

- Hypoglycaemic index: Similar in concept to the Hyperglycaemic index presented in (Vogelzang et al., 2004), defined as the area between the $2.6 \mathrm{mmol} / \mathrm{L}$ threshold and the CGM trace (for CGM trace $<2.6 \mathrm{mmol} / \mathrm{L}$ ) summed over the entire length of stay, normalised by the length of data record. Note: the units used in this study are $\mu \mathrm{mol} / \mathrm{L}$, not $\mathrm{mmol} / \mathrm{L}$ as in (Vogelzang et al., 2004). 


\section{RESULTS}

An example of the modified slope parameter after recalibration compared to original slope parameter is shown in Figure 2 for a representative CGM record.

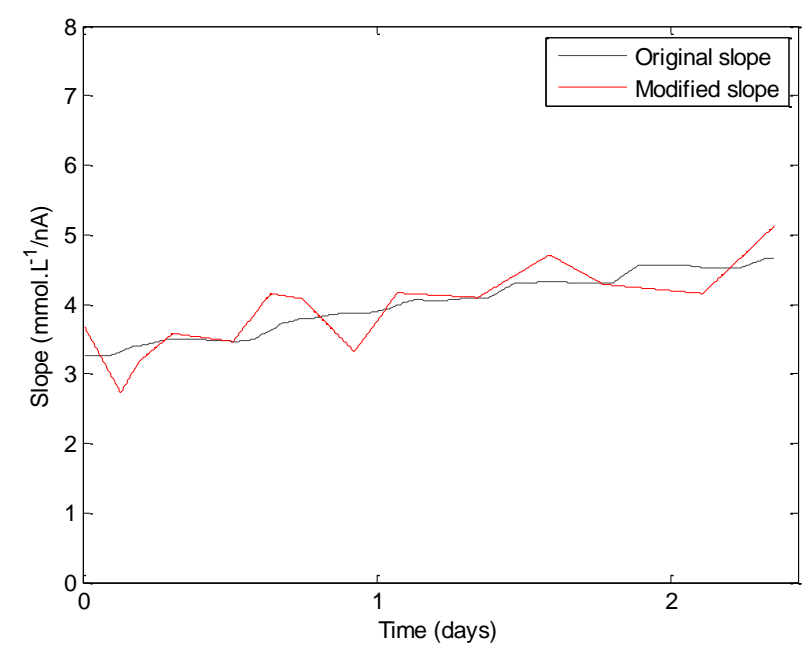

Figure 2: Example of the modification to the slope parameter when re-calibrating.

Figure 3 shows the distribution of CGM errors. CGM error is defined as the CGM glucose minus the BG measurement, at the time of calibration. The top plot shows the error distribution for all of the CGM-BG data (1074 pairs). The middle plot shows the distribution of errors for the CGM-BG data where either the CGM measurement or the BG measurement is less than or equal to $3 \mathrm{mmol} / \mathrm{L}$ ( 145 pairs). The bottom plot shows the distribution of errors for the CGM-BG data where either the CGM measurement or the BG measurement is greater than $7 \mathrm{mmol} / \mathrm{L}$ (62 pairs). In all 3 plots the dashed vertical line represents the median, and the solid vertical lines represent the interquartile range (IQR).

Figure 4 shows a section of CGM trace comparing original (black dashed line), recalibrated (solid blue line),
Recalibrated and filtered (green dotted line), and Filtered Valid_I IIG then recalibrated variations (purple dash-dot line). In this example, overall trends in calibration parameters and CGM output are preserved. However it is clear in Figure 4 that metrics of hypoglycaemia will vary for each method of signal processing.

Table 3 compares the number, duration and severity of hypoglycaemia events, as well as the hypoglycaemic index for each variation of the CGM calibration. The results are presented for the overall cohort and per-patient to show any potential skewed results from individual patients.

Table 4 shows the number of patients who gained, lost or remained (the same) with/without hypoglycaemia after recalibration and filtering, where the middle two rows indicate patients who changed status.
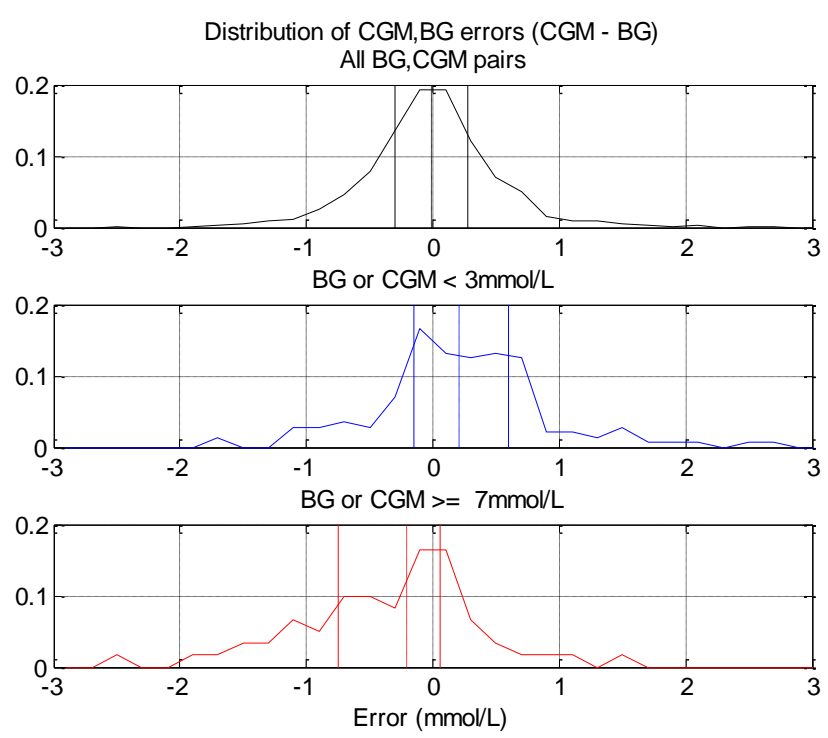

Figure 3: Distribution of errors between CGM and BG measurements with median (dashed vertical line) and interquartile range (solid vertical lines), for different glucose levels.

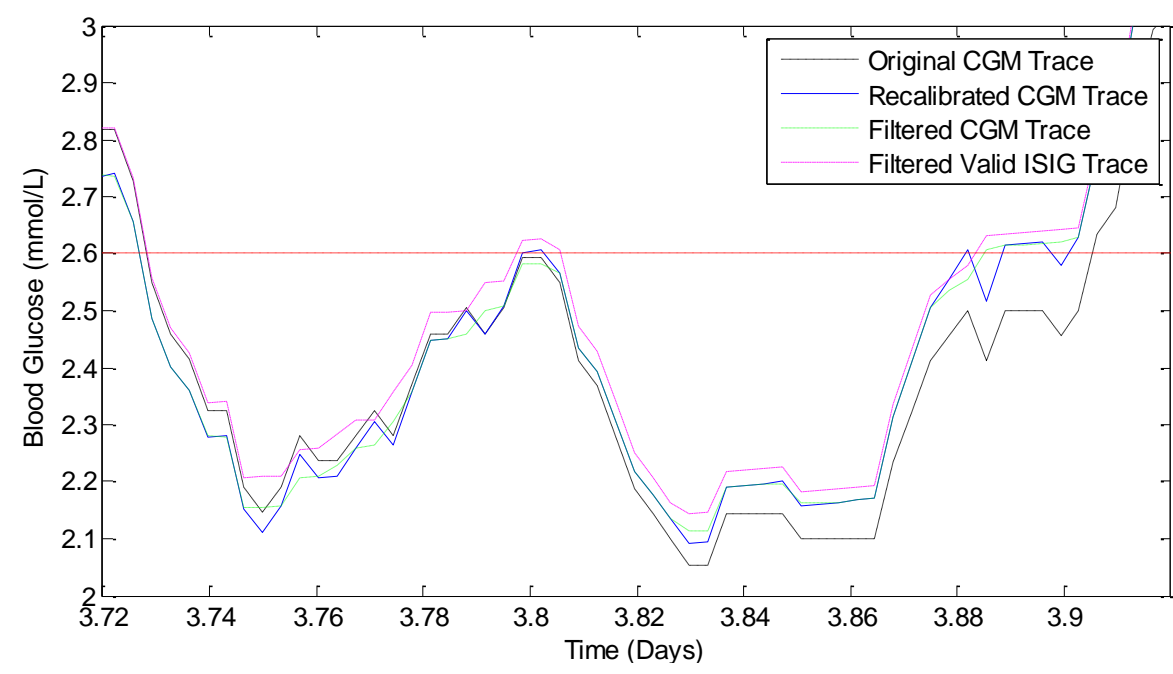

Figure 4: Comparison of a section of CGM trace containing hypoglycaemia for Original CGM, Recalibrated CGM, Recalibrated and Filtered CGM, and Filtered ISIG then Recalibrated. 
Table 3: Effect of recalibration and filtering on recorded CGM hypoglycaemia for the entire cohort and per-patient. Results are presented as Median [Inter-quartile range] where applicable.

\begin{tabular}{|c|c|c|c|c|}
\hline Overall cohort results & Original CGM data & Re-calibrated CGM & Re-calibrated and filtered CGM & Filtered ISIG and Re-calibrated CGM \\
\hline Number of Hypoglycaemic events & 161 & 193 & 131 & 146 \\
\hline Duration (\% of CGM record $<2.6 \mathrm{mmol} / \mathrm{L}$ ) & 2.2 & 2.6 & 2.5 & 2.6 \\
\hline Hypoglycaemic index ( $\mu \mathrm{mol} / \mathrm{L})$ & 4.9 & 7.1 & 6.9 & 6.8 \\
\hline Hypoglycaemia events between $2.2-2.4 \mathrm{mmol} / \mathrm{L}$ & 35 & 40 & 35 & 34 \\
\hline Hypoglycaemia events between $2.0-2.2 \mathrm{mmol} / \mathrm{L}$ & 18 & 38 & 23 & 30 \\
\hline Hypoglycaemia events less than $2.0 \mathrm{mmol} / \mathrm{L}$ & 21 & 28 & 22 & 21 \\
\hline \multicolumn{5}{|l|}{ Per-patient results } \\
\hline Number of Hypoglycaemic events & $3[2-7]$ & $3[1-7]$ & $3[1-4]$ & $2[1-4]$ \\
\hline Duration (\% of data hypoglycaemic) & $1.0[0.6-5.9]$ & $2.0[0.8-3.3]$ & $2.0[0.8-3.6]$ & $1.9[0.7-3.3]$ \\
\hline Hypoglycaemic index $(\mu \mathrm{mol} / \mathrm{L})$ & $2.1[0.7-6.8]$ & $4.3[1.0-10.1]$ & $4.3[1.0-10.7]$ & $3.9[0.7-9.5]$ \\
\hline
\end{tabular}

Table 4: Effect of recalibration and filtering on whether a patient gained, lost or stayed with/without hypoglycaemia compared to original CGM data.

\begin{tabular}{|l|c|c|c|}
\hline & Re-calibrating only & Re-calibrating and filtering & Filtering ISIG then Re-calibrating \\
\hline Original hypoglycaemia --> Hypoglycaemia (\# patients) & 24 & 22 \\
\hline Original hypoglycaemia --> No hypoglycaemia (\# patients) & 1 & 23 \\
\hline Originally no hypoglycaemia --> Hypoglycaemia (\# patients) & 8 & 3 \\
\hline Originally no hypoglycaemia --> No hypoglycaemia (\# patients) & 17 & 7 & 18 \\
\hline
\end{tabular}

\section{DISCUSSION}

The aim of this study was to investigate how calibration sensor error and non-linear filtering of CGM data, both together and separately, affect the observed number, duration and severity of hypoglycaemia $(\mathrm{BG}<2.6 \mathrm{mmol} / \mathrm{L})$ in at risk preterm babies. This knowledge is important for accurately relating hypoglycaemia to long term outcomes. CGM data is recalibrated to assess the impact of different, more accurate reference sensor measurements.

Table 3 shows all metrics of hypoglycaemia increased after recalibration, which can potentially be explained by skew in the distribution of $\mathrm{BG}$ vs. CGM readings at low BG concentrations.

Figure 3 shows the distribution of errors between the CGMBG paired measurements. The data set contains 1074 paired BG-CGM measurements of which $51 \%$ have a BG measurement higher than the CGM and $49 \%$ have a BG measurement lower than the CGM, and this is overall relatively centred, as expected from the regression aspect of the calibration algorithm.

More importantly, the second plot in Figure 3 shows a definite positive shift in the median when only considering low glycaemic levels. Of the 145 pairs containing either a CGM or BG measurement below $3 \mathrm{mmol} / \mathrm{L}, 63 \%$ have a BG measurement lower than the CGM. These lower measurements pull the CGM trace down to the more accurate BG analyser value when recalibrating and cause the hypoglycaemia metrics to increase. Additionally, the bottom plot in Figure 3 shows the opposite is also true for high BG concentrations. At least for our dataset, CGM readings have a greater tendency to be lower than their BG counterparts when the concentrations are above $7 \mathrm{mmol} / \mathrm{L}$.

When comparing recalibrated CGM data to recalibrated and filtered CGM data for the overall cohort, the large reduction in the number of observed hypoglycaemic events (193 to $131)$ with very little change in hypoglycaemic duration $(2.6 \%$ to $2.5 \%$ ) can be explained by reference to Figure 4 . There are two different phenomena that reduce the number of events from 4 to 1 in this exemplar case. First, at 3.8 days the peak in the CGM trace is trimmed by the filter (filter after recalibration) stopping it crossing the normoglycaemic threshold and thus reducing the number of hypoglycaemic events. The opposite is also likely to occur, where a hypoglycaemic event observed in the recalibrated CGM trace is removed by filtering (in this case a trough is trimmed). The second phenomenon is seen at $\sim 3.88$ to 3.9 days where high frequency fluctuations in CGM measurements are smoothed by the filter. Smoothing high frequency fluctuations around the threshold is likely to be the major influence on the reduced number of hypoglycaemic events observed. The difference in the number of hypoglycaemic events observed over the 4 variations of CGM data suggests that this metric alone (number of events) may not be reliable when classifying clinical hypoglycaemia.

Table 4 shows the number of patients that gained, lost or stayed with/without hypoglycaemia when recalibrated and filtered, compared to original CGM data. Of the 25 babies who had hypoglycaemia in the original data set 22 to $24 \mathrm{had}$ hypoglycaemia in the modified data sets, and, 17 to 18 out of 25 of babies who had no hypoglycaemia in the original data set still had no hypoglycaemia. These results suggest that over the entire duration of monitoring, the CGM should be 
consistent $\sim 80 \%$ of the time about which patients had experienced hypoglycaemia at some stage, independent of calibration method.

\section{CONCLUSIONS}

The aim of this study was to investigate how recalibrating and filtering CGM data affects the observed number, duration and severity of hypoglycaemia in preterm infants. The results suggest that conventional hypoglycaemia metrics are heavily dependent on both the CGM reference sensor error and the calibration algorithm used. All metrics of hypoglycaemia for our cohort increased after recalibration, confirming that the standard CGM algorithm tended to overestimate BG at lower levels by assuming a higher error, less accurate reference sensor. If highly accurate calibration measurements are available it may be more appropriate to recalibrate the data, especially when trying to accurately classify hypoglycaemia or other specific extreme events.

More importantly and generally, reference sensor error and thus calibration algorithms play a significant role in quantifying hypoglycaemia using CGM data. In particular, metrics such as number of hypoglycaemic events are particularly sensitive to recalibration effects. While this conclusion may be expected, its potential impact is quantified here, in this case for at-risk neonates for whom hypoglycaemia may carry long-term negative consequences.

\section{REFERENCES}

ABBOTT 2010. Abbott Optium Test-Strip Packet Insert. UK: Abbott Diabetes Care Ltd.

CHEE, F., FERNANDO, T., SAVKIN, A. \& VAN HEERDEN, P. 2001. The use of MiniMed CGMS in real-time glucose monitoring. Anziis 2001: Proceedings of the Seventh Australian and New Zealand Intelligent Information Systems Conference, 159-164.

CORNBLATH, M., HAWDON, J. M., WILLIAMS, A. F., AYNSLEY-GREEN, A., WARD-PLATT, M. P., SCHWARTZ, R. \& KALHAN, S. C. 2000.

Controversies regarding definition of neonatal hypoglycemia: suggested operational thresholds. Pediatrics, 105, 1141-5.

HARRIS, D. L., BATTIN, M. R., WESTON, P. J. \& HARDING, J. E. 2010. Continuous Glucose Monitoring in Newborn Babies at Risk of Hypoglycemia. Journal of Pediatrics, 157, 198-202.

KLONOFF, D. C. 2000. The Importance of Continuous Glucose Monitoring in Diabetes. Diabetes Technology \& Therapeutics, 2, S1-S3.

KOH, T. H. H. G., EYRE, J. A. \& AYNSLEYGREEN, A. 1988. Neonatal Hypoglycemia - the Controversy Regarding Definition. Archives of Disease in Childhood, 63, 1386-1388.
MASTROTOTARO, J., GROSS, T. \& SHIN, J. 2002. Glucose monitor calibration methods. United States patent application. Jul 2002.

MINIMED, M. 2003. CGMS System Gold User Guide.

PRETTY, C. G., CHASE, J. G., LE COMPTE, A., SHAW, G. M. \& SIGNAL, M. 2010. Hypoglycemia detection in critical care using continuous glucose monitors: an in silico proof of concept analysis. $J$ Diabetes Sci Technol, 4, 15-24.

ROCHE 2007. ACCU-CHEK Comfort Curve Test-strip packet insert. USA: Roche Diagnostics Ltd.

ROCHE 2008. Evaluation Report of the ACCU-CHEK Comfort Curve Test Strip as a Plasma-like Test Strip. Roche Diagnostics, Mannheim, Germany.

ROSSETTI, P., BONDIA, J., VEHI, J. \& FANELLI, C. G. 2010. Estimating Plasma Glucose from Interstitial Glucose: The Issue of Calibration Algorithms in Commercial Continuous Glucose Monitoring Devices. Sensors, 10, 10936-10952.

SIGNAL, M., PRETTY, C. G., CHASE, J. G., LE COMPTE, A. \& SHAW, G. M. 2010. Continuous glucose monitors and the burden of tight glycemic control in critical care: can they cure the time cost? J Diabetes Sci Technol, 4, 625-35.

SOLNICA, B., NASKALSKI, J. W. \& SIERADZKI, J. 2003. Analytical performance of glucometers used for routine glucose self-monitoring of diabetic patients. Clin Chim Acta, 331, 29-35.

STANLEY, C. A. \& BAKER, L. 1999. The causes of neonatal hypoglycemia. New England Journal of Medicine, 340, 1200-1201.

VOGELZANG, M., HORST, I. C. V. D. \& NIJSTEN, M. W. 2004. Hyperglycaemic Index as a Tool to Assess Glucose Control: A Retrospective Study. Crit Care, 8, R122-R127. 